\title{
Hardware Engineering for an APT in a TEM Objective Lens
}

\section{Brian Gorman}

\section{Colorado School of Mines, Golden, Colorado, United States}

The most widespread APT data reconstruction method was first proposed in 1995 [1]. Incremental changes to the method have lead to improved accuracies as datasets became larger, laser field evaporation became more widespread, and heterogeneous materials systems became more commonplace. Unfortunately, errors during APT data reconstruction are still limiting widespread acceptance of APT as a quantitative imaging technique. There are many factors contributing to these inaccuracies, but they can include inaccurate estimation of specimen geometry based upon the assumed evaporation field, inaccurate assumptions of the ion trajectories, and magnification changes due to varying field evaporation characteristics at heterointerfaces. Utilizing ex-situ correlative TEM imaging with APT has been shown to improve the accuracy of data reconstruction through the quantification of many of the reconstruction variables, including radius, shank angle, atomic spacing, detection efficiency, image compression factor, and field of view [2]. However, the full use of TEM has not yet been applied to the APT experiment as a wealth of other information are useful and readily attainable. Our group has previously engineered a UHV electron microscope on to an existing commercial APT instrument [3], however, the imaging performance was not of high enough spatial resolution to allow for reconstruction improvements. Therefore, in order to further improve APT as a quantitative imaging and analysis technique, hardware enabling in-situ APT within a TEM objective lens is needed [4].

Requirements for APT are significantly more challenging than most commercial TEM objective lenses currently allow. For instance, the specimen must be cooled to cryogenic temperatures nominally $<50 \mathrm{~K}$ for accurate spatial resolution, the specimen must be situated in a UHV environment to prevent surface contamination and mass spectral signal to noise, a local high field must be applied to the specimen to direct the ions, an ion detector must be fitted to the TEM column normal to the specimen axis to measure the field evaporated ions, and, for non-metallic specimens, a focused laser pulse must be applied to the specimen apex to induce field evaporation. The TEM requirements are much more modest and include imaging at $\sim 60,000 \mathrm{x}$ using a $16 \mathrm{Mp}$ camera and a method to capture diffraction patterns relative to the specimen axis.

Our hardware solution [5] enables APT within a TEM objective lens using a modest investment of capital hardware. UHV is obtained using a combination of turbomolecular pumping and a $\sim 4 \mathrm{~K}$ cryogenic trap within the TEM pole gap. Cryogenic specimen temperatures are achieved using a recirculating cryocooler with vacuum transfer to both the side entry stage and the objective lens cryo trap. A commercially available large field of view delay line ion detector assembly fit to a UHV flange is engineered to bolt on the TEM column opposite the specimen holder. Laser integration with commercial in-situ TEM hardware are currently available and being utilized for this instrument.

Electron imaging and diffraction are utilized in a correlative manner for quantification of the specimen function before the field evaporation experiment. The specimen function contains the real space volume and atom positions (determined from reciprocal space) and can subsequently be created and sliced perpendicular to the evaporation axis. Comparing the specimen function with ion field evaporation maps from the correlative APT experiment in Fourier space allow for quantification of aberrations during the field evaporation experiment. As such, APT may one day become a simple addition to existing electron microscopy hardware. 


\section{References}

[1] P. Bas, A. Bostel, B. Deconihout, D. Blavette, Applied Surface Science, 87-88, 298-304 (1995).

[2] D. R. Diercks, B. P. Gorman, Ultramicroscopy 195, 32 (2018).

[3] R. Kirchhofer, D. R. Diercks, B. P. Gorman, Review of Scientific Instruments, 89, 053706 (2018).

[4] T. Kelly, M. Miller, K. Rajan, S. Ringer, A. Borisevich, N. Dellby, O. Krivanek, Microscopy and Microanalysis, 17(S2), 708-709 (2011).

[5] B. P. Gorman, US Patent \#16/384,427, April 16, 2019. 